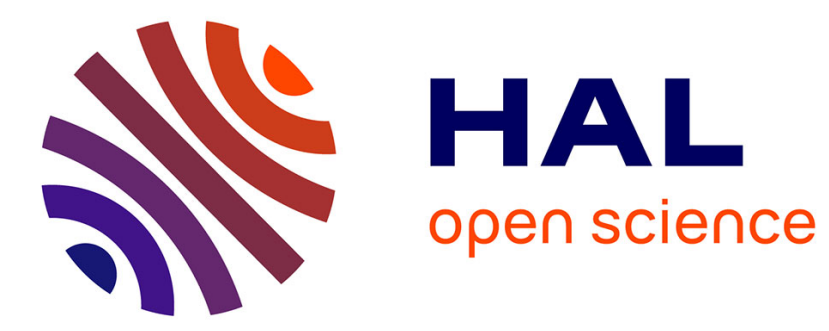

\title{
Living Lab MACVIA-LR Fragilité
}

M. Nogues, Claude Jeandel, Jacques Touchon, N. Pinto, Hubert Blain, M. Léglise, D. Paccard, V. Bruguière, A.-L. Coupet, D. Marc, et al.

\section{To cite this version:}

M. Nogues, Claude Jeandel, Jacques Touchon, N. Pinto, Hubert Blain, et al.. Living Lab MACVIA-LR Fragilité. La Presse Médicale, 2015, 44, pp.S36 - S46. 10.1016/j.lpm.2015.07.010 . hal-01753910

\section{HAL Id: hal-01753910 https://hal.science/hal-01753910}

Submitted on 8 Nov 2018

HAL is a multi-disciplinary open access archive for the deposit and dissemination of scientific research documents, whether they are published or not. The documents may come from teaching and research institutions in France or abroad, or from public or private research centers.
L'archive ouverte pluridisciplinaire HAL, est destinée au dépôt et à la diffusion de documents scientifiques de niveau recherche, publiés ou non, émanant des établissements d'enseignement et de recherche français ou étrangers, des laboratoires publics ou privés. 


\title{
Living Lab MACVIA-LR Fragilité
}

\author{
M. Noguès ${ }^{1,12}$, C. Jeandel ${ }^{2,3,12}$, J. Touchon ${ }^{3,4}$, N. Pinto $^{1}$, H. Blain ${ }^{2,3}$, M. Léglise $^{2}$, D. Paccard ${ }^{1}$, V. Bruguière ${ }^{1}$,

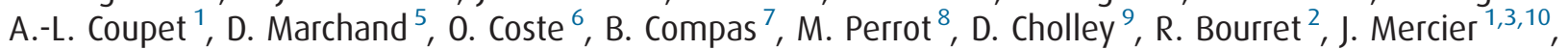 \\ T. Camuzat ${ }^{7}$, J.-P. Michel ${ }^{11}$, J. Bousquet ${ }^{2}$, M. Marc $^{1}$, J.-C. Reuzeau ${ }^{1}$
}

1. Institut régional du vieillissement, Carsat Languedoc-Roussillon, 34000 Montpellier, France

2. CHU, 34000 Montpellier, France

3. Université Montpellier I, 34000 Montpellier, France

4. Inserm U1061, neuropsychiatrie : recherche épidémiologique et clinique, 34000 Montpellier, France

5. Agence régionale de santé Languedoc-Roussillon (ARS), 34000 Montpellier, France

6. Directions régionales de la jeunesse, des sports et de la cohésion sociale (DRJSCS), 34000 Montpellier, France

7. Conseil régional Languedoc-Roussillon, 34000 Montpellier, France

8. Régime social des indépendants (RSI), 34000 Montpellier, France

9. Direction régionale du service médical (DRSM), 34000 Montpellier, France

10. Inserm U1046, physiologie et médecine expérimentale, cœur et muscles, université Montpellier I et II, 34000 Montpellier, France

11. Université de Genève, European Union Geriatric Medicine Society (EUGMS), Genève, Suisse

Correspondance :

Jean Bousquet, CHRU de Montpellier, 34295 Montpellier cedex 5, France.

jean.bousquet@orange.fr fragilité, situation dont la fréquence augmente avec l'avancée en âge, constitue un problème médical, social et sociétal majeur. Elle doit par conséquent être détectée et prise en charge rapidement afin de prévenir ou de retarder la dépendance. MACVIA-LR aborde ce problème selon une approche holistique et dans un continuum intégrant les risques de fragilité et les stades pré-fragilité et fragilité. Ce projet, en cours de déploiement, s'appuie sur des dispositifs intégrés, modélisant un authentique Living Lab, et sur une gouvernance territoriale préfigurant les coordinations des prototypes " parcours de santé des personnes âgées en risque de perte d'autonomie »

12 Les deux auteurs ont participé également à la rédaction de l'article.
(PAERPA). Les deux approches représentent des projets intégrés et en réseau sur le modèle des Living Labs.

\section{Situation du problème. Justification du modèle Living Lab pour les sujets âgés fragiles}

Le repérage précoce de la fragilité chez les personnes âgées a pour objectif d'identifier les déterminants de la fragilité et d'agir sur eux afin de retarder la perte d'autonomie dite " évitable » et ainsi de prévenir la survenue d'événements défavorables. En amont de la fragilité observée, le repérage des risques de fragilité peut apporter une vision plus globale sur la population susceptible de bénéficier d'un accompagnement adapté, tant sur le champ somatique que dans le domaine social. 


\section{Le phénotype fragilité : un état potentiellement réversible}

La Société française de gériatrie et de gérontologie (SFGG) a adopté en 2011 [1] la définition suivante de la fragilité : « Lo fragilité est un syndrome clinique. II reflète une diminution des capacités physiologiques de réserve qui altère les mécanismes d'adaptation au stress. Son expression clinique est modulée par les comorbidités et des facteurs psychologiques, socioux, économiques et comportementoux. Le syndrome de fragilité est un marqueur de risque de mortalité et d'événements péjoratifs, notamment d'incapacités, de chutes, d'hospitalisation et d'entrée en institution. L'âge est un déterminant majeur de fragilité mais n'explique pas à lui seul ce syndrome. La prise en charge des déterminants de la fragilité peut réduire ou retarder ses conséquences. Ainsi, la fragilité s'inscrirait dans un processus potentiellement réversible ».

\section{Deux modèles de critères de fragilité validés}

Un modèle fondé sur un phénotype " physique " qui évalue la présence chez les personnes d'un âge $\geq 65$ ans de 5 critères : perte de poids involontaire de plus de $4,5 \mathrm{~kg}$ (ou $\geq 5 \%$ du poids) depuis 1 an, épuisement ressenti par le patient, vitesse de marche ralentie, baisse de la force musculaire et sédentarité. Les patients sont dits fragiles en présence de 3 critères ou plus. Ils sont dits "pré-fragiles » si au moins un des critères est présent. Si aucun des critères n'est constaté, ils sont considérés comme robustes [2].

Un autre modèle de fragilité prend en compte des critères fondés sur l'intégration de facteurs physiques, cognitifs et sociaux, regroupés sous le terme de " fragilité multi-domaine » intégrant : cognition, humeur, motivation, motricité, équilibre, capacités pour les activités de la vie quotidienne, nutrition, condition sociale et comorbidités [3].

Le Living Lab MACVIA-LR fragilité s'inspire de ce modèle en abordant le concept de fragilité, à la fois dans ses dimensions sociales, psycho-cognitives et physiques. Les déterminants sociaux de la fragilité sont importants à considérer [4-6],

\section{Glossaire}

ARS Agence régionale de santé

CARSAT Caisse d'assurance retraite et de la santé au travail

CESAF Centre d'évaluation des sujets âgés fragiles

CNAV Caisse nationale assurance vieillesse

CNAMTS Caisse nationale d'assurance maladie des travailleurs salariés

COPEX Comité de pilotage et d'experts

IRV Institut régional du vieillissement

MAIA Maisons pour l'autonomie et l'intégration des malades Alzheimer

PAERPA parcours de santé des personnes âgées en risque de perte d'autonomie puisqu'ils interagissent fréquemment avec les autres déterminants, physiques, psychologiques et cognitifs. En outre, le Living Lab intègre une démarche holistique fondée sur des facteurs de risque repérables soit à un niveau individuel, soit à un niveau populationnel.

\section{Le phénotype fragilité : un marqueur prédictif de perte d'autonomie, de difficultés de mobilité, de chute, d'hospitalisation et de décès}

Le repérage de la fragilité permet de prédire le risque de perte d'autonomie (niveau de preuve élevé), de chutes, d'institutionnalisation, de décès et d'hospitalisation des personnes âgées de 65 ans ou plus (niveau de preuve modéré), dans un délai de 1 à 3 ans. La réversibilité de la fragilité n'est pas en règle générale spontanée et nécessite le plus souvent des interventions. Des interventions gériatriques fondées sur l'évaluation gériatrique standardisée (EGS) et la coopération avec les acteurs des soins primaires peuvent réduire le risque d'hospitalisation des sujets âgés fragiles. L'obtention d'un bénéfice sur les risques de dépendance, de déclin fonctionnel et d'institutionnalisation est également possible (niveau de preuve modéré). D'autres bénéfices potentiels peuvent découler du repérage de la fragilité : l'adaptation de la prise en charge des comorbidités et l'ajustement de la prise en charge en cas d'hospitalisation. Inversement, il peut permettre à des sujets qui ne sont pas fragiles d'accéder à certains soins qui leur sont parfois refusés du fait de leur âge.

\section{Population cible : un repérage opportuniste après 70 ans mais une attention à porter dès la survenue des premiers facteurs de risque chez les seniors plus jeunes}

La prévalence de la fragilité dépend de la définition utilisée. Cela est souligné par l'analyse de la littérature avec une prévalence moyenne de $10 \%$, mais avec des variations qui peuvent aller de 5 à $58 \%$. Dans l'étude SHARE, réalisée dans 10 pays européens, la prévalence de la fragilité selon le phénotype de Fried a été évaluée pour la France à 15,5\% parmi les sujets âgés de plus de 65 ans vivant à domicile [7]. Cette prévalence augmente avec l'âge et elle est plus importante chez les femmes. En considérant le ratio entre la prévalence de la fragilité et les bénéfices potentiels des interventions, on peut proposer comme champ du repérage les personnes âgées de plus de 70 ans, indemnes de maladie grave, sans dépendance avérée, et à l'initiative d'un soignant soupçonnant une fragilité.

Dans le champ plus large de l'approche des risques de fragilité, le croisement de données issues des systèmes d'information de la Sécurité sociale (maladie et retraite) montre des situations de renoncement aux soins, d'isolement, de précarité qui sont autant de facteurs prédictifs d'une évolution vers une fragilité avérée. Une prise de conscience précoce de ces facteurs permet de mettre en place un plan d'action s'inscrivant dans un concept élargi de prévention sociale (travaux des observatoires des situations de fragilité, CNAMTS 2013). 


\section{Le Living Lab MACVIA-LR}

Il s'appuie sur une gouvernance régionale des risques de fragilité, I'Institut régional du vieillissement, communauté animée par la Caisse d'assurance retraite et de la santé au travail (Carsat), et sur une gouvernance territoriale de la fragilité avérée préfigurant les coordinations des prototypes " parcours de santé des personnes âgées en risque de perte d'autonomie (PAERPA) 》 et intégrant les structures de type MAIA (Maisons pour l'autonomie et l'intégration des malades Alzheimer).

Il est original car il déploie une approche multidisciplinaire pour repérer et agir sur les déterminants de la fragilité. Cette approche implique entre autres les acteurs des secteurs sociaux, médico-sociaux et sanitaires sur l'expertise gériatrique et I'ensemble des acteurs MACVIA-LR [8].

\section{Une gouvernance régionale des risques de fragilité impliquant la Carsat Languedoc-Roussillon et I'Institut régional du vieillissement \\ La Carsat Languedoc-Roussillon}

Il s'agit d'un organisme de Sécurité Sociale à compétence régionale. Structure de droit privé exerçant une mission de service public, elle intervient auprès des salariés, des retraités et des entreprises de la région, au titre de la retraite, de l'action sociale et de la gestion des risques professionnels (figure 1).
La Carsat du Languedoc-Roussillon regroupe plus de 800 salariés dont 220 dans les services décentralisés départementaux (agences retraite et service social). Elle est au service de 100000 employeurs, 563000 salariés actifs et 493000 retraités. Elle a trois missions :

- mettre à jour le compte retraite tout au long de la carrière, préparer et verser la retraite des salariés ;

- assurer et prévenir les risques professionnels dans les entreprises ;

- accompagner les assurés en difficulté : la Carsat aide les personnes fragilisées par la maladie, le handicap ou l'accident et met en œuvre des réponses sociales adaptées aux besoins des personnes. Elle favorise le maintien à domicile des personnes fragilisées mais encore autonomes.

La Carsat représente : 12 agences de proximité ; 24000 personnes reçues en agence et points d'accueil ; 3 milliards d'euros versés dans l'économie régionale ; 19000 personnes bénéficiant d'un accompagnement du service social, 60 sessions de prévention et de promotion de la santé, 753000 heures pour l'aide à domicile.

\section{L'Institut régional du vieillissement \\ Fondements}

Dans le champ de l'accompagnement des assurés en difficulté, I'Institut régional du vieillissement (IRV) a été créé à l'initiative

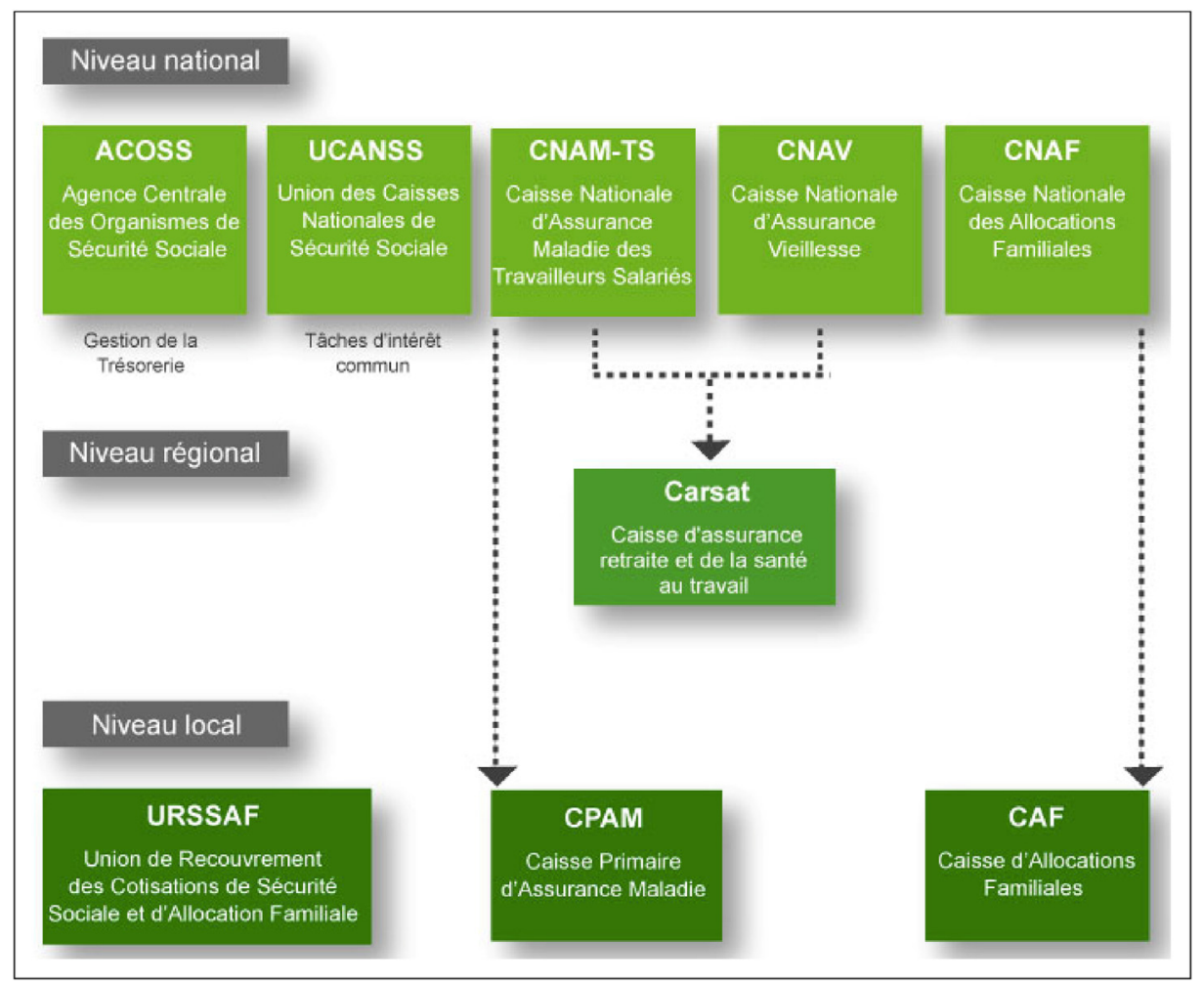

FIGURE 1

La Carsat dans la protection sociale française 
de la Carsat du Languedoc-Roussillon. Il s'agit de positionner la Carsat au sein d'une communauté, dans l'environnement institutionnel et partenarial régional à l'égard des assurés sociaux retraités en situation de fragilité. L'IRV vise à fédérer et à regrouper, au sein d'une même unité, différentes activités dispensées par les services de la Carsat, le but étant la coordination et la création de parcours attentionnés en faveur des personnes âgées fragiles. La création de cet Institut du vieillissement a donné lieu à différents échanges, d'une part avec les autres régimes qui seront appelés à contribuer à son activité et d'autre part, avec les partenaires essentiels que sont notamment l'ARS et les conseils généraux.

Le management opérationnel de l'IRV est assuré par un Comité de pilotage et d'experts (COPEX). Le COPEX qui recouvre différents partenaires (ARS, DRJSCS, CG, CPAM, RSI/MSA, CHU...) a été élargi aux Présidents des Commissions Assurance Maladie, Action Sociale et Innovation et Prospective de la Carsat.

Le COPEX est appelé notamment à déterminer les axes de développement de I'IRV. Sur un plan formel, I'IRV est porteur des actions à déployer en région au titre du Plan national dit « Proximité Autonomie ", promu conjointement par la CNAMTS et la CNAV. L'IRV regroupe différentes activités.

Un pôle " observation "

Afin de mener une politique de proximité ciblée en matière de prévention, la Carsat-LR a mis en œuvre un Observatoire sur les situations de fragilité élargi à l'Inter-régime (Mutualité Sociale Agricole - Régime Social des Indépendants [MSA - RSI]) [9]. Cet Observatoire vise à repérer les risques de fragilité des assurés pour promouvoir des actions de prévention précoces et adaptées aux besoins des personnes pour maintien de leur autonomie et l'identification des territoires prioritaires d'intervention afin de développer des actions concertées au niveau local. Les informations sont accessibles sur le site de la Carsat-LR et elles peuvent être consultées par l'intermédiaire d'un Système d'information géographique (SIG).

Ces informations permettent d'établir un diagnostic de fragilité territoriale défini sur la base d'un indicateur composite prenant en considération des facteurs de risques économiques et d'isolement social. Les indicateurs sont issus des bases de données de la Carsat, de l'Inter-régime (MSA - RSI) et du réseau Assurance Maladie du régime général (CPAM - Direction régionale du service médical). Les offres d'actions collectives de prévention des risques du vieillissement répertoriées sont celles conduites et/ou financées par I'Inter-régime et I'ARS.

Le travail d'observation est essentiel afin de mieux connaître les populations âgées en repérant particulièrement les risques de fragilité. L'Observatoire des situations de fragilité mis en place par la Carsat constitue aujourd'hui un outil inter-régime qui fait partie intégrante de I'IRV.

Par la suite, l'Observatoire sera amené à capitaliser en cohorte les informations nécessaires au suivi des actions et permettre ainsi leur évaluation.
Un pôle " labellisation et évaluation "

L'IRV évalue les activités des acteurs de proximité et des prestataires de services auprès des personnes âgées, en insistant sur la coordination. Le but est de créer un environnement favorable qui contribue à une prise en charge globale dans les meilleures conditions possibles. En l'absence de réels gestionnaires des situations individuelles de type "gestionnaire en prévention sociale ", il s'agit de favoriser les échanges autour de la personne âgée.

L'IRV est appelé à labelliser les structures qui contribuent à cet objectif et à évaluer sous l'angle commun de la « qualité » et de " l'utilité » les actions engagées. Ce dispositif vient en appui de « l'appel à projets régional et inter-régime » porté par la Carsat, l'inter-régime et ses partenaires, en apportant une appréciation qualitative sur les porteurs de projet et les actions qu'ils souhaitent mettre en œuvre.

Les organismes de Protection sociale du Languedoc-Roussillon et I'ARS se rassemblent autour d'un appel à projets commun pour prévenir les risques liés au vieillissement des seniors fragilisés. Des actions et des "recherches/expérimentations " seront financées sur les territoires prioritaires d'intervention définis par l'Observatoire des situations de fragilité. Pour 2014, est attendue une enveloppe disponible de plus de $400000 €$.

Un pôle offre de services en "guichet concerté "

Autour de l'Espace Seniors de la Carsat, l'IRV intègre un « guichet concerté " préconisé par le Plan national proximité autonomie (circulaire CNAMTS/CNAV du 20/09/2014) [10]. II vise à apporter aux assurés sociaux retraités ou préretraités en risque de fragilité, des conseils et des orientations dans le domaine de la retraite, mais aussi sur le champ de la santé avec la participation des autres régimes, des organismes de retraite complémentaire et des mutuelles.

Le « guichet concerté » [11] fait appel aux compétences existantes (conseiller retraite, technicien de l'Assurance maladie...). Il permet de repérer les personnes en risque de fragilité, d'après une grille d'appréciation de la fragilité réalisée avec le concours d'experts, telles qu'elles peuvent être signalées par l'Observatoire mais aussi par les Services Retraite, les Services Sociaux, la plateforme téléphonique de la Carsat, les pôles évaluateurs et/ou les prestataires de services soit par l'interrégime, soit par tout autre partenaire, afin de leur apporter des conseils et de favoriser leur orientation. Des liens à cet égard sont établis avec des centres de prévention (centre de prévention "Montpastel » du régime Agirc/Arrco, plateforme d'évaluation du département de gériatrie du CHU de Montpellier. . .). Les personnes signalées pourront être ainsi orientées vers des activités de prévention.

Ce schéma propre à l'Espace Seniors de Montpellier sera progressivement étendu à l'ensemble de la région à partir des agences "Retraite » de la Carsat. À cet égard, des liens seront à tisser avec les Centres d'examen de santé de l'Assurance Maladie. 
Un pôle " Recherche, études et expérimentations »

L'Institut du vieillissement intégrera un espace dédié aux nouvelles technologies. Est envisagée, à ce titre, la mise à disposition d'une tablette tactile s'inscrivant dans un objectif double de préservation du lien social et d'une meilleure coordination des intervenants, ce projet rejoignant celui à déployer par la Carsat dans le cadre des "paniers de services » de l'Action Sociale. En complément, est envisagée la mise à disposition de bracelets intelligents ou de jeux intelligents.

En outre, un suivi de cohorte est prévu afin d'identifier les évolutions en matière de fragilité pour ce qui concerne la population dotée.

À cet égard, il s'agira de procéder à une analyse multimodale des risques de fragilité avec une définition des profils de fragilité et le suivi dynamique d'une cohorte à partir de graphiques « radars » ou grille permettant le repérage des risques de fragilité dans quatre dimensions : moteur, psychosocial, nutritionnel et cognitif (figure 2).

Dans ce cadre, une réflexion a été engagée visant à faire évoluer le modèle conceptuel de la prévention sur le champ spécifique des effets du vieillissement. Le modèle conceptuel de la prévention repose habituellement sur trois niveaux (prévention primaire, secondaire, tertiaire). Ceux-ci revêtent une réelle utilité dès lors qu'il s'agit de repérer les actions à mener et d'une certaine manière de les classer par nature mais surtout par typologie de vecteurs de communication et d'actions. L'usage de ces niveaux peut conduire à des segmentations qui permettent difficilement d'avoir une approche globale à l'égard des personnes et ce d'autant plus lorsque les sujets sont âgés et surtout lorsqu'ils ont des risques de fragilité. La prévention des effets du vieillissement impose de revoir les modes de communication et d'approche de cette population afin de concevoir des réponses parfaitement adaptées.

À la formulation classique des trois niveaux de prévention (primaire, secondaire, tertiaire), il paraît préférable de revisiter le concept par un changement de paradigme qui reviendrait à revoir les approches préventives en les fondant sur une

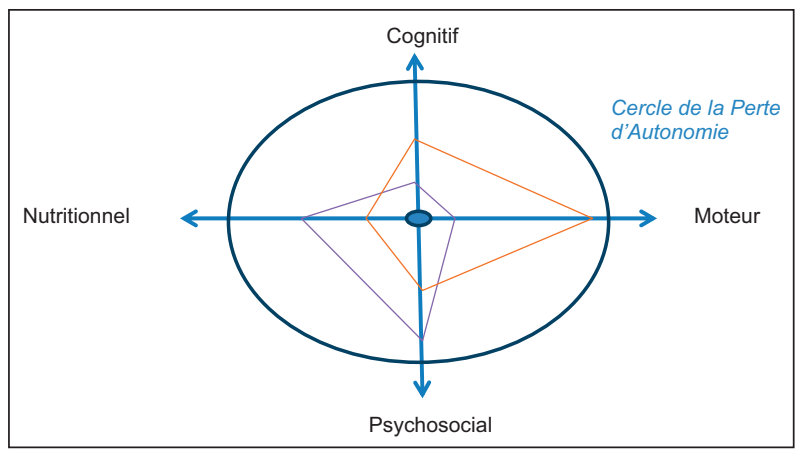

FIGURE 2

Exemple de profil différentiation entre les domaines concernés par la fragilité, à savoir (figure 3) :

- l'état de santé et la maladie selon la définition de l'OMS ;

- la santé sociale en s'appuyant sur la représentation qu'à la personne de son champ personnel d'activité ;

- l'environnement socio-économique qui prend en compte la personne dans un environnement plus élargi qui est de fait son autonomie sociale.

Cette nouvelle approche de la démarche de prévention des effets du vieillissement permettrait d'appréhender les réponses qui pourraient être apportées par rapport aux risques de fragilité perçus, tout en conservant une dimension globale par rapport à la question essentielle de la préservation de l'autonomie.

\section{Une gouvernance territoriale de la fragilité avérée préfigurant les coordinations des prototypes PAERPA assurant le dispositif opérationnel du Living Lab}

Sur un plan plus opérationnel, le Living Lab est situé sur le territoire de la coordination territoriale d'appui (CTA) du prototype parcours de santé des PAERPA [12].

Ce dispositif est actuellement en cours de déploiement sur le territoire Est-héraultais au sein duquel la CTA est assurée par la MAIA Est-héraultaise (dispositif d'intégration des services de soins et d'accompagnement pour les personnes âgées en perte d'autonomie).

Ce territoire correspond

- aux 44 communes de l'agglomération de Montpellier ;

- au territoire d'intervention du CLIC Maillage ;

- au territoire d'intervention de l'UTAG (unité territoriale d'action gérontologique) Est-héraultaise du Conseil Général 34.

Cette plateforme, basée sur le concept de l'intégration [6], est destinée aux personnes âgées fragiles (ou PAERPA) et à leurs aidants, et surtout aux professionnels des trois secteurs qui les prennent en charge, pour optimiser leurs parcours de santé.

Ce dispositif a pour avantage de pouvoir s'appuyer sur les organes et instruments de la MAIA :

- les deux tables de concertation : stratégique (gouvernance du projet, composée des décideurs et financeurs) et tactique (acteurs et services fédérés) ;

- le guichet intégré ;

- la gestion de cas ;

- I'annuaire opérationnel des ressources.

Pour rappel, les fonctions de ce niveau de coordination sont les suivantes (cf. rapport et cahier des charges PAERPA) et visent à atteindre le niveau de l'intégration [13] :

- information et orientation vers l'ensemble des ressources socio-sanitaires disponibles sur le territoire ;

- activation des aides sociales pour les équipes de proximité et les établissements de santé ;

- une expertise gériatrique en interne ou en mobilisant la(les) filière(s) gériatrique(s) de proximité ; 


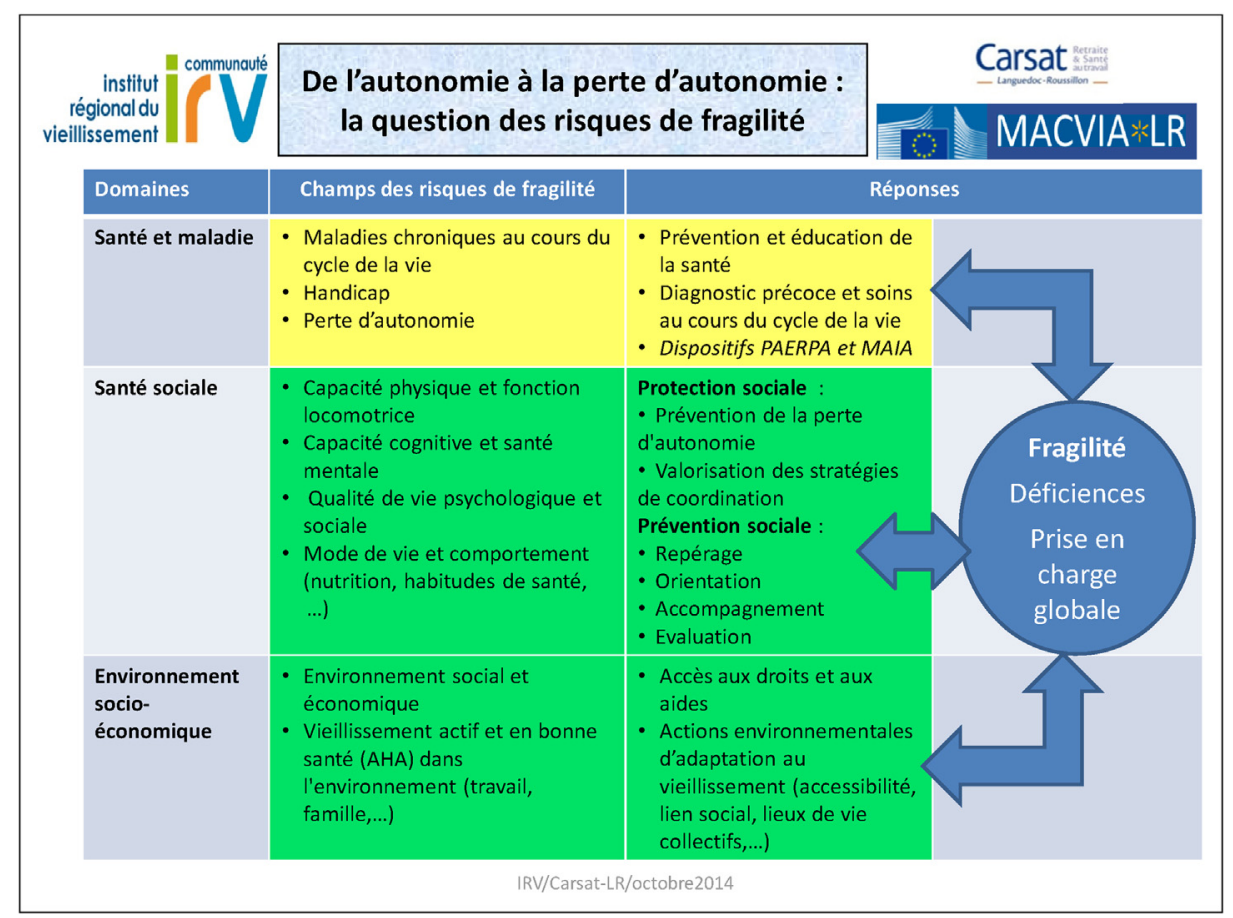

\section{FIgURE 3}

Nouvelle approche de la démarche préventive

- rôle social et sanitaire à jouer dans l'élaboration du PPS, mais sans démobiliser les acteurs de proximité ;

- la gouvernance de la coordination sur le territoire.

\section{La structure de gouvernance territoriale : les deux tables de concertation de la MAIA}

En conformité avec la réglementation, la MAIA dispose d'une table stratégique mise en œuvre par l'Agence régionale de santé (ARS LR) chargée d'identifier les besoins, les ressources, et de jouer un rôle d'alerte et de planification, et d'une table tactique mise en œuvre par les professionnels de terrain, une représentation de tous les professionnels des trois secteurs concernés (notamment gériatres et hospitaliers) ainsi que des PAERPA et des aidants.

\section{Une démarche en trois actes Repérer - Évaluer - pour, Agir en impliquant l'ensemble des acteurs Repérer}

Le repérage de la fragilité ne doit être envisagé et réalisé que comme la première étape d'une séquence comprenant l'évaluation globale de la personne et la planification d'interventions visant à prévenir la perte d'autonomie, les chutes, l'institutionnalisation et l'hospitalisation (figure 4).

Ce repérage recours à un questionnaire comportant 7 questions, utilisable par les acteurs impliqués [14] (figure 5). En tant que de besoin, il est souhaitable que les personnes identifiées soient orientées vers leur médecin traitant qui complétera ce questionnaire afin de prendre ou non la décision de recourir à la plateforme d'évaluation du Centre d'évaluation des sujets âgés fragiles (CESAF) du CHU de Montpellier.

Le repérage peut être réalisé par le médecin traitant ou par un autre soignant de premier recours : infirmière, pharmacien, kinésithérapeute, aide-soignante, etc.

L'implication des services sociaux et d'aide à la personne dans le repérage est un élément majeur : la détection précoce des signes de fragilité par les travailleurs sociaux qui réalisent des évaluations en réponse aux demandes d'APA et par les aide-ménagères au domicile, grâce à l'utilisation d'outils adaptés, est une voie à explorer et à évaluer.

Évaluer

L'évaluation, fondée sur l'EGS, peut être réalisée en hôpital de jour gériatrique ou en ambulatoire, en recourant à une équipe mobile gériatrique, à un réseau de santé de personnes âgées, etc. Elle doit s'attacher à évaluer les comorbidités, l'environnement social et économique qui modulent l'expression de la fragilité (figure 4). Elle doit conduire à des interventions « multi-domaines » de prévention qui peuvent porter sur :

- I'activité physique adaptée et la lutte contre la sédentarité ;

- la nutrition ;

- la réduction de la polymédication et l'optimisation thérapeutique :

- la mise en place d'aides sociales, l'adaptation de l'environnement et la mobilisation des liens sociaux. 


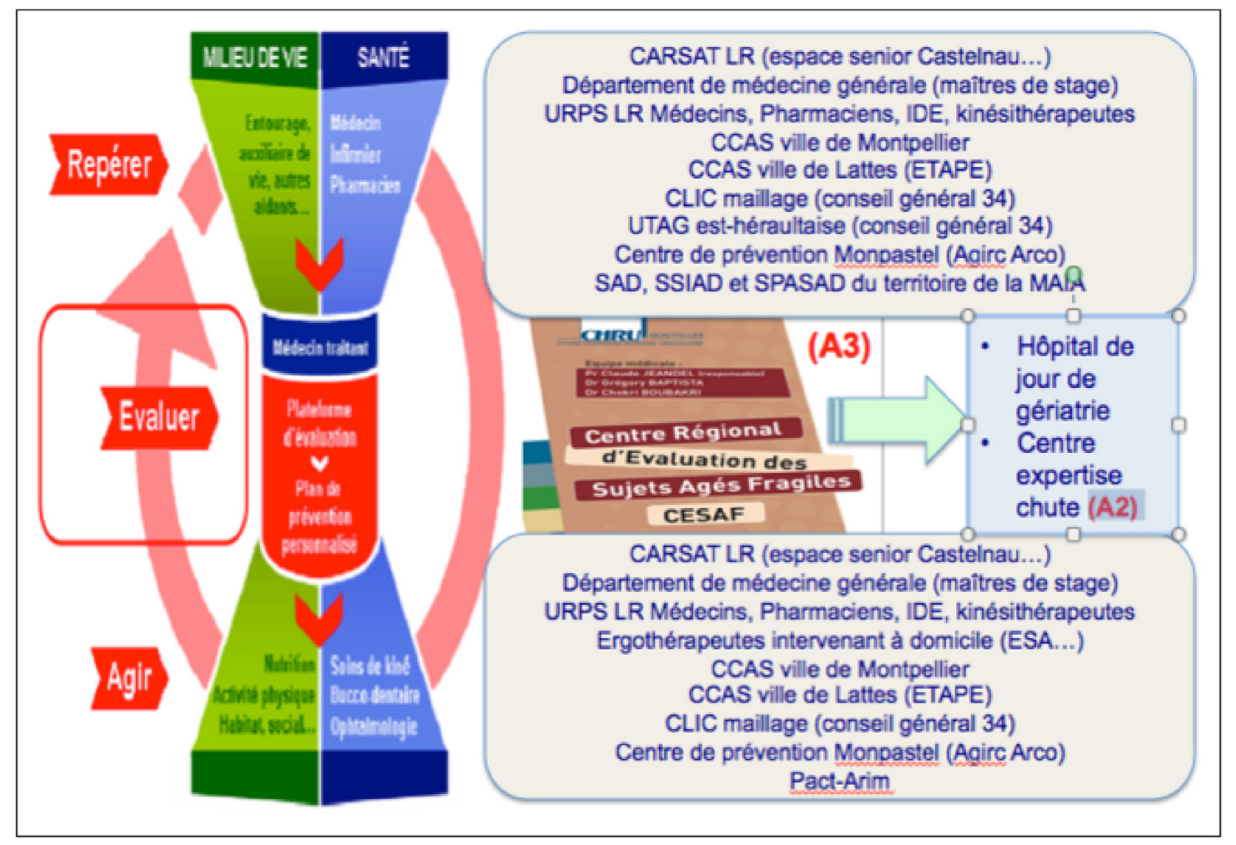

FIGURE 4

Les trois étapes : Repérer, Évaluer, Agir et les acteurs impliqués

Les acteurs et partenaires du Living Lab impliqués dans l'évaluation du sujet âgé fragile :

- le Centre d'évaluation des sujets âgés fragiles (CESAF) du CHU de Montpellier ;

- le Centre de prévention Monpastel (AGIRC ARCO).
Le CESAF. Le pôle de gérontologie du CHU de Montpellier met à la disposition des médecins généralistes, un centre d'évaluation des sujets âgés fragiles et de prévention de la dépendance, qui procède à des interventions médicales, paramédicales et/ou sociales (en lien avec une équipe pluri-professionnelle :

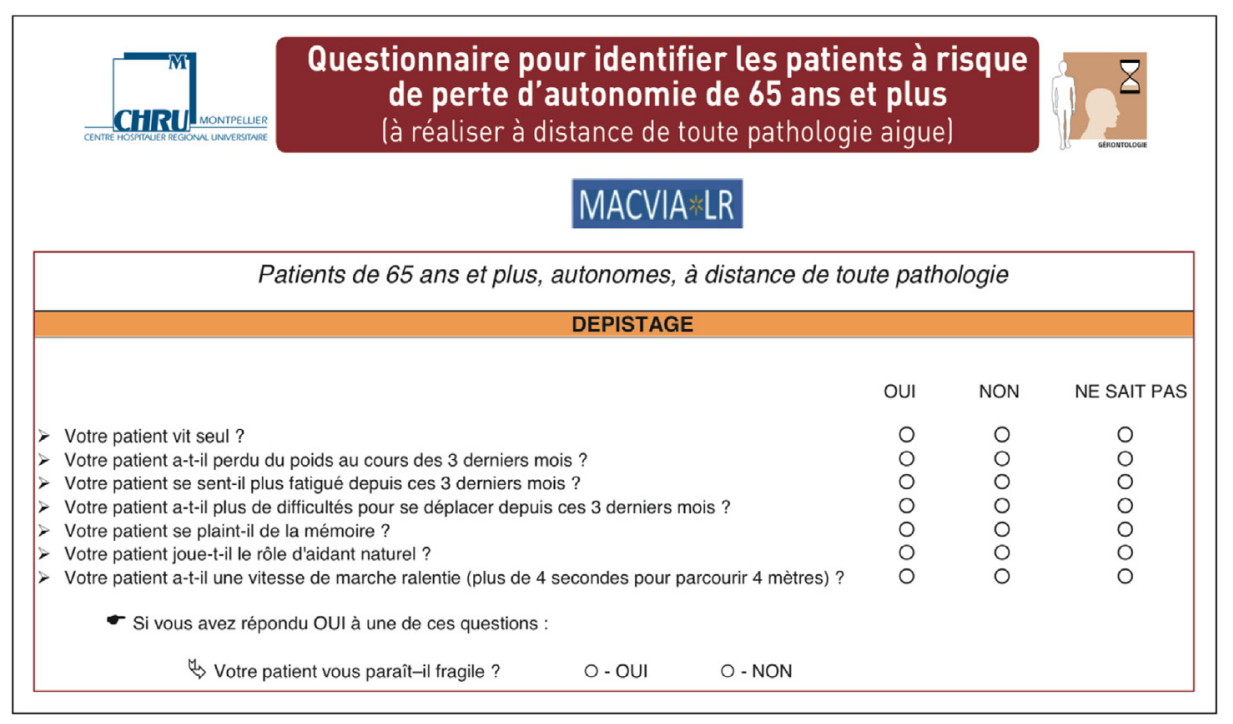

FIGURE 5

Questionnaire pour le repérage de la fragilité chez les plus de 65 ans 
psychologues, kinésithérapeutes, diététiciens...), en collaboration étroite avec les médecins traitants et les professionnels de terrain. Son objectif principal est de retarder le processus d'entrée dans la dépendance des personnes âgées fragiles et pré-fragiles par :

- la catégorisation du sujet âgé dans l'un des trois phénotypes : robuste, fragile/pré-fragile, dépendant ;

- I'identification des critères de la fragilité ;

- la recherche des déterminants de la fragilité : médicaux, sociaux, environnementaux, économiques. .., par une évaluation pluri-professionnelle ;

- I'élaboration d'un plan d'intervention personnalisé pour prévenir la dépendance en fonction des causes retrouvées ;

- accessoirement, l'accès à l'innovation et à la recherche clinique dans le cadre de protocoles de recherche axés sur la prévention de la dépendance.

Les différentes étapes mises en œuvre :

- mise en place d'aide à la décision pour les médecins généralistes à partir d'une évaluation gériatrique pluri-professionnelle : le test de dépistage doit conduire à la réalisation d'une évaluation gériatrique multidimensionnelle par une équipe pluridisciplinaire permettant de confirmer le diagnostic de "fragilité » et d'en définir les principaux éléments qui la composent. Ceux-ci servent de base à l'élaboration d'une intervention personnalisée de soins. Cette évaluation gériatrique est réalisée par un médecin gériatre ou un médecin généraliste formé à l'utilisation et l'interprétation des principaux tests dans un délai court (moins d'une semaine) ;

- prévention de la dépendance : au terme de cette évaluation multiparamétrique, un plan d'intervention est élaboré. II est personnalisé et adapté à la situation clinique du patient. Il peut associer des interventions médicales (consultations spécialisées), paramédicales (neuropsychologues, kinésithérapeutes, diététiciennes...) et/ou sociales. Il est coordonné par le médecin généraliste traitant, articulé avec les acteurs de terrain. Pour chaque patient, un dossier d'évaluation contenant une grille d'évaluation et un plan personnalisé de prévention est préparé. Un suivi au long terme sera organisé par l'équipe du centre, en lien étroit avec le médecin généraliste.
Le Centre de prévention Monpastel procède également à une évaluation à l'issue de laquelle il peut être conduit à orienter les personnes âgées vers le CESAF avec lequel il a été établi une convention.

\section{Agir}

Ces interventions doivent être formalisées dans le cadre d'un plan personnalisé de prévention. La réalisation de l'évaluation et des interventions en ambulatoire pour la plupart des patients suppose de développer l'offre collective de prévention par les acteurs des soins primaires. Un repérage négatif doit être répété si la situation du patient se modifie (événement de vie, pathologie incidente, etc.).

Acteurs et partenaires du Living Lab impliqués dans le repérage et les actions inscrites dans le plan personnalisé de prévention :

- la Carsat Languedoc-Roussillon et son Espace Seniors (Castelnau le Lez);

- les médecins maîtres de stage (encadrant les internes de médecine générale) du département de médecine générale de I'UFR de médecine ;

- les médecins généralistes du territoire de la MAIA représentés par I'URPS LR ML et leurs associations de FMC (FMC 34) ;

- les pharmaciens du territoire de la MAIA représentés par I'URPS LR des pharmaciens ;

- les kinésithérapeutes du territoire de la MAIA représentés par I'URPS LR des kinésithérapeutes ;

- les ergothérapeutes des équipes spécialisées Alzheimer (ESA) et ergothérapeutes intervenant au domicile ;

- les IDE du territoire du territoire de la MAIA représentés par I'URPS LR IDE ;

- le CCAS de la ville de Montpellier (membre de la table tactique de la MAIA et en convention avec le CESAF);

- le CCAS de la ville de Lattes et l'ETAPE de Lattes (membre de la table tactique de la MAIA et en convention avec le (ESAF);

- le Centre de prévention Monpastel AGIRC ARCO (en convention avec le (ESAF);

- les services d'aides à domicile (SAD) et de soins infirmiers à domicile (SSIAD/SPASAD) fédérés au sein du transi-SSIAD du territoire de la MAIA ;

- le PACT-ARIM.

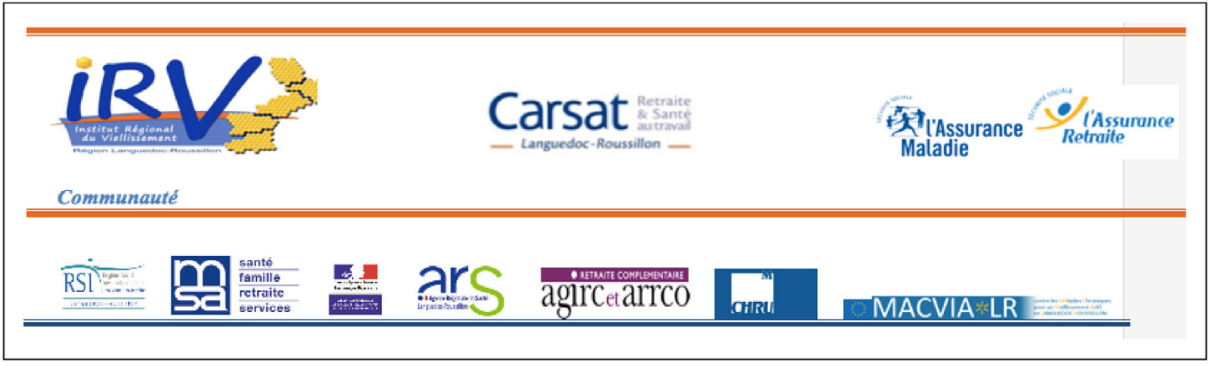

FIGURE 6

Les partenaires de l'Institut régional du vieillissement 

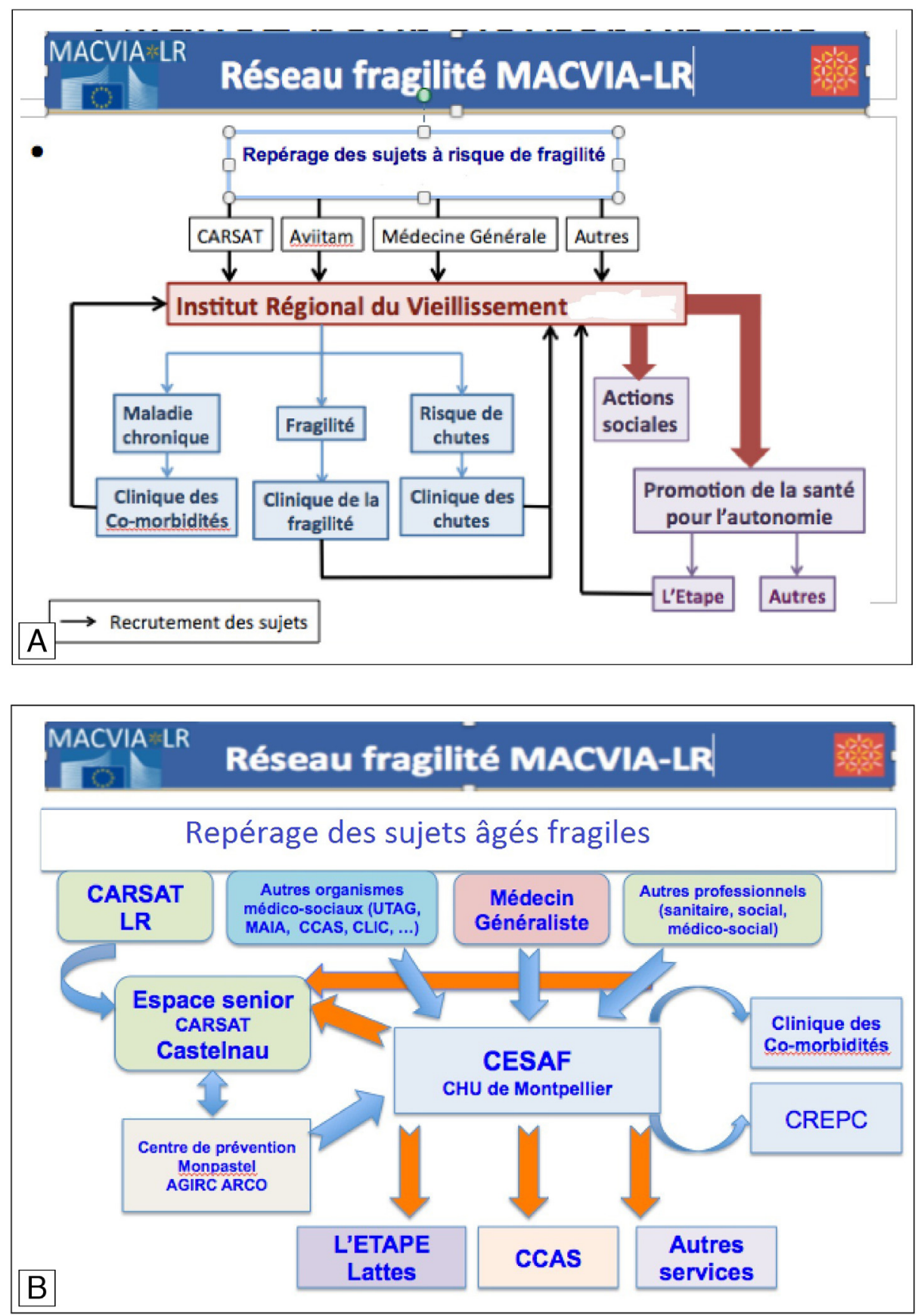

FIGURE 7

A. Réseau fragilité MACVIA-LR : le dispositif opérationnel à l'échelon régional (risques de fragilité). B. Réseau fragilité MACVIA-LR : le dispositif opérationnel à l'échelon territorial (fragilité avérée)

CARSAT : Caisse d'assurance retraite et de la santé au travail ; CCAS : Centre action sociale ; CESAF : Centre régional d'évaluation des sujets âgés fragiles ; CREPC : Centre régional équilibre et prévention des chutes. 
Ces organismes dispensent ou orientent les personnes âgées fragiles en se référant à un annuaire recensant les activités ou prestations proposées aux personnes de plus de 65 ans : club des aînés pour une lutte contre l'isolement ; ateliers mémoires pour la prévention du déclin cognitif ; activités sportives pour lutter contre la dynapénie ; structures d'Aide aux aidants afin de limiter leurs difficultés; centres d'ergothérapie pour lutter contre le handicap et prévenir les risques d'un domicile maladapté ; services de transport pour lutter contre l'isolement ; services d'aide à la personne pour lutter contre l'isolement, le handicap, pour soulager les aidants; services de portage de repas à domicile pour lutter contre la dénutrition.

\section{Les formations}

\section{Formations universitaires}

Premièrement, le démonstrateur Trans-Innov-Longévité (TIL) UM1, Paris VI, UNF3S.

La formation des acteurs impliqués peut recourir au projet TransInnov Longévité (TIL) de I'UNF3S, labellisé « Initiatives d'excellence en formations innovantes » (IDEFI) par le ministère de l'Enseignement supérieur et de la Recherche et porté par l'université Montpellier 1 et l'université Pierre-et-Marie-Curie ParisVI [15]. Le projet TIL modélise un dispositif innovant de formations inter-universitaires transversales adaptées tant aux formations initiales qu'aux formations tout au long de la vie. II démultiplie un système de formations d'excellence s'appuyant sur la potentialisation de réseaux universitaires nationaux et internationaux, à l'instar de celui de l'UNF3S.

Trois diplômes sont ouverts depuis 2014/2015 dans le cadre de ce démonstrateur :

- le Master e-learning de gérontologie (UM1, Paris VI, UNF3S) ;

- le DU Vulnérabilité et fragilité : identification et prise en charge des personnes handicapées et des personnes âgées (université Montpellier 1/IRTS) ;

- le DIU Fragilité et Parcours de santé des personnes âgées en risque de perte d'autonomie (PAERPA) (université Montpellier 1).

Deuxièmement, le Master présentiel de gérontologie (université Montpellier 1).

\section{Formations assurées par I'IRV}

Afin de parvenir à l'objectif d'une prise en charge globale, pertinente, efficace à l'égard des personnes en situation de fragilité, il convient d'accentuer le professionnalisme des différents partenaires de terrain. Il est envisagé de participer à la mise en place de diplômes qualifiants. Un diplôme universitaire (DU) portant notamment sur la prévention sociale, la conception de projets et la fragilité des personnes âgées, sera créé en liaison avec l'ARS, les universitaires et les professionnels concernés. Ce programme de formation est en cours de construction avec l'ARS afin de créer un Diplôme universitaire : " DU gestionnaire de parcours » avec deux options "Parcours en santé » et "Prévention ». Le démarrage de cette formation sera effectif en septembre 2015.

Des séances d'information/formation à destination des partenaires seront mises en place sous le couvert d'une animation par l'Espace Seniors et/ou transposables. Elles concerneront l'accès aux droits et les ciblages populationnels mais également l'évaluation. D'autres séances d'information pourront être développées en fonction de besoins spécifiques (passeport, formation de l'appel à projets...).

Enfin, et dans le temps, pourrait être envisagée la mise en place d'un pôle de formateurs dédiés.

\section{Intégration dans le projet MACVIA-LR et perspectives}

L'IRV MACVIA-LR est sous l'égide de partenaires publics (figure 6).

Sous cette présentation, I'Institut du vieillissement est opérationnel et c'est au fil de sa mise en place que de nouveaux segments de contribution à l'accompagnement de personnes âgées apparaîtront.

Le partenariat avec le projet MACVIA-LR porté par la Région LR et le CHU est un contexte "porteur » de l'approche sur le vieillissement actif. Une intégration dans le projet MACVIA-LR est mise en place [16-19]. Elle regroupe les différents acteurs régionaux qui ont une action de prévention et/ou de traitement de la fragilité (figure $7 a$ et $b$ ).

D'ores et déjà, I'IRV a été positionné comme Living Lab sur la fragilité et est en relation à ce titre avec différents partenaires potentiels œuvrant également pour le développement d'une approche " globale » des risques de fragilité dans l'avancée en âge.

Déclaration d'intérêts : les auteurs déclarent ne pas avoir de conflits d'intérêts en relation avec cet article.

\section{Références}

[1] Rolland Y, Benetos A, Gentric A, Ankri J, Blanchard F, Bonnefoy $M$, et al. Frailty in older population: a brief position paper from the French Society of Geriatrics and Gerontology. Geriatr Psychol Neuropsychiatr Vieil 2011;9:387-90.

[2] Fried LP, Ferrucci L, Darer J, Williamson JD, Anderson G. Untangling the concepts of disability, frailty, and comorbidity: implications for improved targeting and care. J Gerontol A Biol Sci Med Sci 2004;59:255-63.

[3] Rockwood K, Song X, Macknight C, Bergman $\mathrm{H}$, Hogan DB, McDowell I, et al. A global clinical measure of fitness and frailty in elderly people. CMAJ 2005;173:489-95.
[4] Gobbens RJ, van Assen MA, Luijkx KG, Wijnen-Sponselee MT, Schols JM. Determinants of frailty. J Am Med Direct Assoc 2010;11:356-64

[5] Andrew MK, Keefe JM. Social vulnerability from a social ecology perspective: a cohort study of older adults from the National 
Population Health Survey of Canada. BMC Geriatrics 2014;14:90.

[6] Salem BE, Nyamathi A, Phillips LR, Mentes JC, Sarkisian C, Brecht ML. Development of a frailty framework among vulnerable populations. ANS Adv Nurs Sci 2014;37:70-81.

[7] Santos-Eggimann B, Cuenoud P, Spagnoli J, junod J. Prevalence of frailty in middle-aged and older community-dwelling Europeans living in 10 countries. J Gerontol A Biol Sci Med Sci 2009:64:675-81.

[8] Bousquet J, Bourquin C, Augé P, Domy P, Bringer J, Camuzat T, et al. MACVIA-LR reference site of the European Innovation Partnership on Active and Healthy Ageing. Eur Geriatr Med 2014;5:406-15.

[9] CNAMTS/CNAV. Atlas des situations de fragilité 2014. https://www.lassuranceretraite.fr/ cs/Satellite/atlas-situations-fragilite.pdf? blobkey=idgblobwhere $=52889167046158$ blobcol=urldata\&blobtable=MungoBlo ; 2014 [Accès au site le 30/03/2015].

[10] CNAMTS/CNAV. Circulaire sur le plan Proximité Autonomie de l'Avancée en Âge (P3A) 20 septembre 2014. https://www.partenairesactionsociale.fr/accueil/contenu.do?
actionStruts=/ppas/portal/GAAD GRP_ARCH_N/GAAD_PG_AN_Documentation/ GAAD PG AN Textes nationaux.html : 2014 [Accès au site le 30/03/2015].

[11] Le guichet concerté dans le cadre de I'IRV, Expérimentation, octobre 2014 Carsat LR. https://www.carsat-Ir.fr/telechargements/ pdf/pdf_general/ra2013.pdf ; 2014 [Accès au site le 30/03/2015].

[12] Libault D, Legrain S, Jeandel C, Burnel P, Von Lennep F. Parcours de santé des personnes âgées en risque de perte d'autonomie. Rapport sur la mise en œuvre des projets pilotes. Paris: IGAS; 2013.

[13] Somme D, Trouvé H, Passadori Y, Corvez A, Jeandel C, Bloch MA, et al. The French Society of Geriatrics and Gerontology position paper on the concept of integration. Int I Integr Care 2014; 14:e052.

[14] HAS. Comment repérer la fragilité en soins ambulatoires. Recommandation de la Haute Autorité de santé (HAS). http://www.hassante.fr/portail/jcms/c_1602970/fr/ comment-reperer-la-fragilite-en-soins-ambulatoires ; 2013 [Accès au site le 30/03/2015].
[15] Jeandel C, Belmin I, Spector M, Benhamou AC Mater 2 Gérontologie. http://master2geronto.etud.univ-montp1.fr/nouveau-le-master-idefi-til-expertise-en-gerontologie 2015 [Accès au site le 30/03/2015].

[16] Bousquet J, Hajjam J, Piette F, et al. [The French reference sites of the European Innovation Partnership on active and healthy ageing]. Presse Med 2013;42:1558-61.

[17] Bousquet J, Addis A, Adcock I, et al. Integrated care pathways for airway diseases (AIRWAYS-ICPS). EUr Respir ) 2014;44:304-23.

[18] Bousquet J, Jorgensen C, Dauzat M, Cesario A, Camuzat T, Bourret R, et al. System medicine approaches for the definition of complex phenotypes in chronic diseases and ageing. From concept to implementation and policies. Curr Pharm Des 2014;20: 5928-44.

[19] Blain H, Abecassis F, Adnet P, et al. Living Lab Falls-MACVIA-LR: the falls prevention initiative of the European Innovation Partnership on Active and Healthy Ageing (EIP on AHA) in Languedoc Roussillon. Eur Geriatr Med 2014;5:416-25. 